\title{
In-situ TEM Investigation of Lithiation and Sodiation of 2D Metal Sulfides
}

Maosen $\mathrm{Fu}^{1}, \mathrm{Xiao} \mathrm{Ma}^{1}$ and Dong $\mathrm{Su}^{2}$

${ }^{1}$ Northwestern Polytechnical University, xi'an, Shaanxi, China (People's Republic), ${ }^{2}$ Brookhaven National Lab, Upton, New York, United States

Two-dimensional (2D) metal sulfides show great promise for their potential applications as electrode materials of lithium/sodium ion-batteries because of the weak interlayer van der Waals interactions, which allow the reversible accommodation and extraction of lithium/sodium ions. Here we investigated the lithiation/sodiation pathway of titanium disulfide using in situ TEM combined with synchrotron-based pair distribution function measurement and first-principles calculations

A 2D intercalation reaction proceeds along with the transition from van der Waals interaction between Ti$\mathrm{S}$ slabs to the covalent bonding of S-Li-S, with no symmetry broken. Further lithiation triggers unconventionally multiple step conversion reactions as proved: $\mathrm{LiTiS}_{2} \rightarrow \mathrm{TiS} \rightarrow \mathrm{Ti}_{2} \mathrm{~S} \rightarrow \mathrm{Ti}$. The conversion reaction pathway is also verified in fully discharged sample in coin-cell. The expanded conversion chemistry is supposed to increase the capacity of $\mathrm{TiS}_{2}$ electrode and downgrade the cyclability, whereas the existence of intermediate phases shows the promise of improving the reversibility with a successful control of the state of charge.

During the sodium-ion intercalation process, we observed multiple intermediate phases (phase II, phase $\mathrm{Ib}$, and phase Ia), different from its lithiation counterpart, with varied sodium occupation sites and interlayer stacking sequences. Further insertion of Na ions prompted a multistep extrusion reaction, which led to the phase separation of Ti metal from the Na2S matrix, with its 2D morphology expanded to a 3D morphology. In contrast to regular conversion electrodes, TiS2 still maintained a compact structure after a full sodiation. First-principles calculations reveal that the as-identified phases are thermodynamically preferred at corresponding intercalation/extrusion stages compared to other possible phases. The present work provides the fundamental mechanistic understanding of the lithiation/sodiation process of 2D transition metal sulfides and offers mechanistic insights for designing high-performance electrode materials for LIBs/NIBs. 


\section{a}
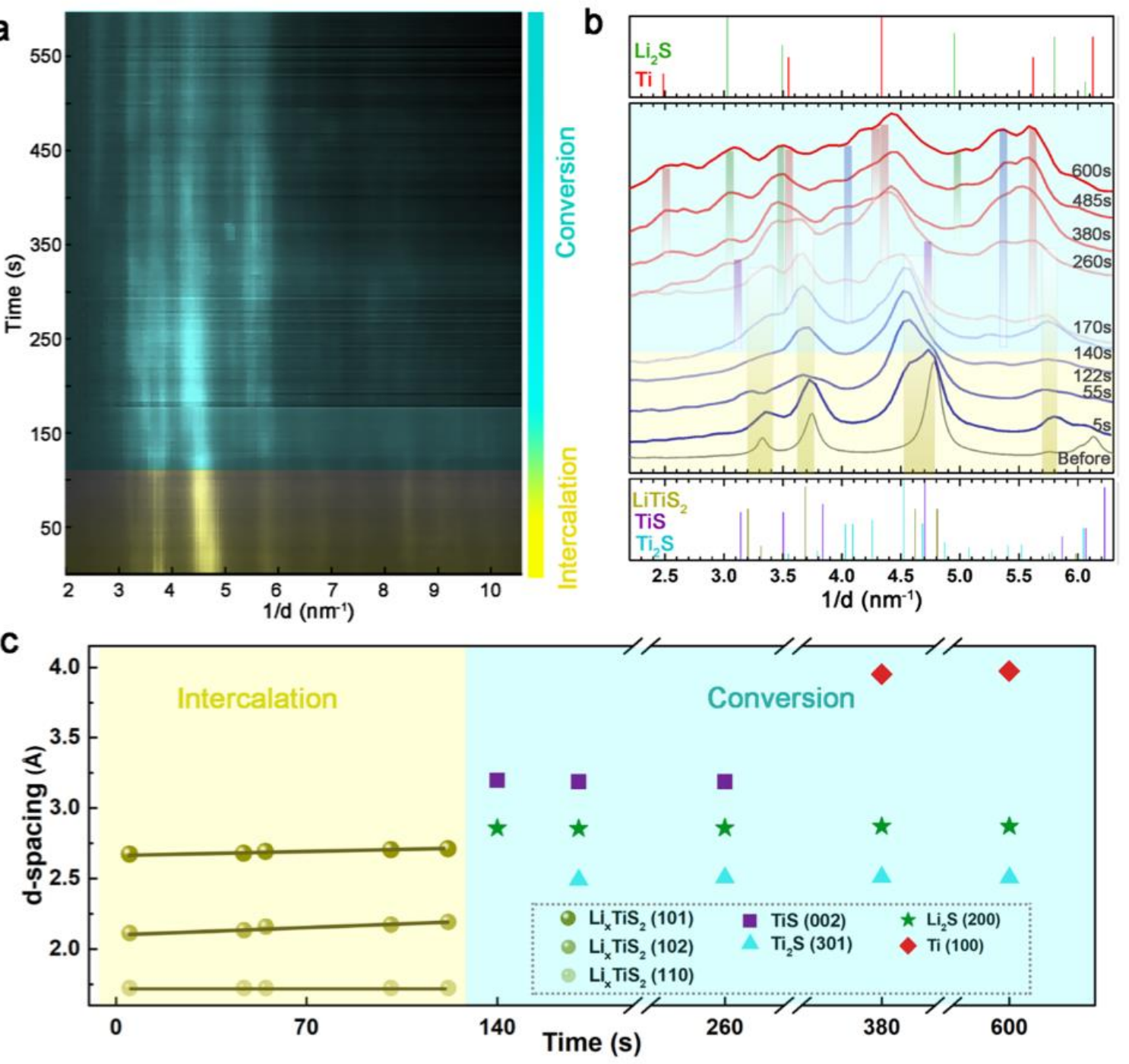

Figure 1. Phase evolution during lithiation probed by in situ electron diffraction. (a) Electron diffraction intensity profile as a function of reaction time during an in situ lithiation of TiS2 plates. (b) Radial intensity profiles of diffraction patterns at certain times. (c) Lattice constant changes measured from SAED during intercalation and conversion. 

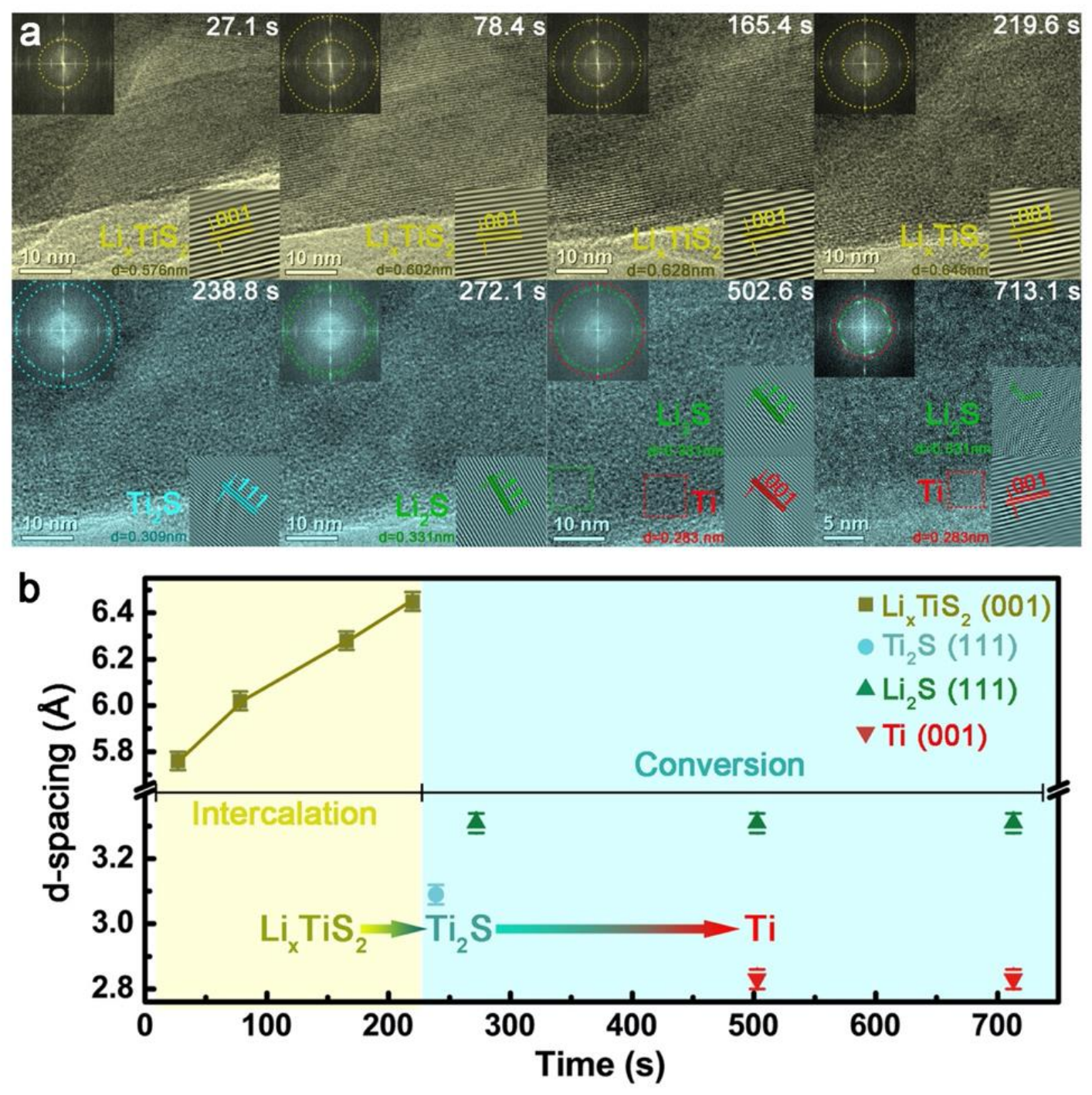

Figure 2. Atomic resolution in situ TEM study. (a) HRTEM images in yellow indicating expansion of LiTiS2 during in situ lithium intercalation and HRTEM images in green revealing existence of Ti2S, Li2S and Ti during in situ lithium conversion. Insets showing FFT patterns and enlarged filtered HRTEM images. (b) Lattice constants measured from LixTiS2 (001), Ti2S (111), Li2S (111) and Ti (001) reflections indicating the intercalation and conversion reactions.

References

Nano Energy 63, 103882 (2019)

ACS Nano 13, 9421-9430 (2019) 